\title{
MODELANDO A Ceratitis capitata (DIPTERA: THEPRITIDAE) PARA ECUADOR.
}

\section{MODELLING TO Ceratitis capitata (Diptera: THEPRITIDAE) FOR ECUADOR}

\author{
García Rosero Magali ${ }^{1}$, Soplín Villacorta Hugo ${ }^{2}$, Alegre Orihuela Julio ${ }^{2}$, Rodríguez Berrio Alexander ${ }^{2}$, \\ Canto Sáenz Manuel ${ }^{2}$, Veneros Guevara Jaris ${ }^{3}$, Vilatuña Rodríguez José ${ }^{4}$, Salas Mujica David ${ }^{4}$ \\ Universidad Nacional Agraria La Molina ${ }^{1}$ \\ Av. La Molina s/n La Molina \\ Lima-Perú \\ 20140685@1amolina.edu.pe ${ }^{1}$ \\ ${ }^{2}$ Profesor Principal, Universidad Nacional Agraria La Molina. \\ ${ }^{3}$ Investigador-Consultor, Universidad Nacional de Cajamarca. \\ ${ }^{4}$ AGROCALIDAD - Programa Nacional del Manejo de Mosca de la Fruta
}

\begin{abstract}
Resumen
Se determinó la distribución potencial de C. capitata bajo condiciones del clima actual y un escenario de cambio climático (A1B-2030) para el Ecuador, empleando el modelo CLIMEX versión 3. El modelo usó parámetros fisiológicos de C. capitata y datos meteorológicos globales para construir un Índice Ecoclimático (IE), el cual describió zonas muy favorables (IE>30), zonas menos favorables (IE<30) y zonas nulas $(I E=0)$ para la distribución de C. capitata durante todo el año. Se estimó que el porcentaje de áreas muy favorables (IE>30) de C. capitata es $47.73 \%$ en condiciones actuales de clima y $33.3 \%$ en el escenario A1B-2030. Adicionalmente, se estimó que el porcentaje de áreas menos favorables (IE<30) es $47.61 \%$ en condiciones actuales de clima y $62.17 \%$ en el escenario A1B-2030. Las áreas de probabilidad nula para establecimiento de C. capitata es $4.65 \%$ para condiciones actuales de clima y 4.80\% para el escenario A1B-2030.
\end{abstract}

Palabras clave: mosca de la fruta, índice ecoclimático, distribución potencial, modelo Climex, escenario de cambio climático

\begin{abstract}
This study determined the potential distribution of $C$. capitata under current climate conditions and a climate change scenario (A1B-2030) for the Ecuador using the CLIMEX model version 3. The model used physiological parameters of $C$. capitata and global meteorological data, to build an Ecoclimatic Index (EI), which described the very favorable areas (EI > 30), less favorable areas (EI < 30) and less favorable areas $(E I=0)$ for the distribution of $C$. capitata throughout the year. It was estimated that the percentage of very favorable areas (EI > 30) of C. capitata is $47.73 \%$ in current climate conditions and $33.3 \%$ in the scenario (A1B-2030). In addition, it was estimated that the percentage of less favorable areas $(E I<30)$ is $47.61 \%$ in current climate conditions and $62.17 \%$ in the scenario $(A 1 B-2030)$. The areas with zero probability for establishment of $C$. capitata is $4.65 \%$ for current climate conditions and $4.80 \%$ for the scenario (A1B-2030).
\end{abstract}

Keywords: fruit fly, ecoclimatic index, potential distribution Climex model, climate change scenario 


\section{Introducción}

La mosca mediterránea de la fruta (moscamed), Ceratitis capitata (Wiedemann) se originó en África subsahariana; es una de las plagas de frutales más destructivas del mundo y posee cerca de 300 hospederos, debido a su amplia distribución, capacidad de tolerar temperaturas frías y de adaptarse a gran variedad de climas, mejor que otras especies de moscas de la fruta. Esta especie ocupa el primer lugar en importancia económica entre las especies de moscas de la fruta [8].Causa daño significativo a las frutas y vegetales, y sus impactos económicos son sustanciales. Es una plaga cuarentenaria, por lo que, a los países con poblaciones de moscamed se les ha establecido barreras comerciales significativas a sus exportaciones.

APHIS [2], en 1992 estimó que, el costo de cada una de las incursiones de moscamed en los EE.UU. (erradicación y la pérdida de la industria) osciló entre US \$ 300.000 y US \$ 200 millones. Investigaciones realizadas por Siebert y Cooper [10] muestran que, brotes de mosca del Mediterráneo en California durante los últimos 25 años han costado a los contribuyentes cerca de \$500 millones, mientras que el brote de la mosca mediterránea de la región de Tampa Bay de la Florida en 1997 dio como resultado \$25 millones gastados en la erradicación [4].

En Ecuador, los estudios de Ceratitis capitata son muy escasos, lo que no ha permitido hasta el momento obtener información que sirva para la implementación de un programa a nivel nacional que reduzca la acelerada proliferación de Ceratitis capitata y mitigar los riesgos económicos, sociales y ambientales que implica su establecimiento en las zonas productoras del Ecuador.

Según datos obtenidos de AGROCALIDAD [1], Ecuador tiene 11250 ha de producción de mango, cucurbitáceas, papaya, mora, tomate de árbol, uvilla, chirimoya, pitahaya y pimiento, en su mayoría solo para mercado interno por la presencia de moscas de la fruta. Esta producción y las que se desean implementar para exportación, es la que se desea cuidar minimizando el riesgo de pérdidas ocasionadas por Ceratitis capitata.

Esta investigación se realizó bajo la necesidad de generar información sobre la distribución potencial actual y bajo un escenario de cambio climático (A1B-2030) para Ceratitis capitata en el Ecuador. Los resultados a obtenerse servirán para implementar estrategias a través del Programa Nacional de Mosca de la Fruta recientemente creado en el Ecuador por medio de la Agencia Ecuatoriana de Aseguramiento de la Calidad del Agro (AGROCALIDAD).

\section{Materiales y Métodos}

El modelamiento de la distribución potencial de la mosca del mediterráneo C. capitata en Ecuador bajo condiciones del clima actual y un escenario de cambio climático (A1B-2030) se llevó a cabo durante el periodo de Septiembre del 2013 a Febrero 2015. El presente estudio abarcó un ámbito a nivel país (Ecuador) y se desarrolló en las oficinas del Programa Nacional de Manejo de Mosca de la Fruta, ubicado en el edificio MAGAP, Quito, Ecuador.

Uso del modelo CLIMEX versión 3.- El clima es principal factor contrastante de la distribución potencial de muchos organismos [18]. CLIMEX integra las respuestas semanales de una población al clima en una serie de índices anuales. La temperatura, la humedad y horas luz se combinan en un índice semanal de crecimiento de la población (GIW por sus siglas en inglés) para las especies. Las respuestas a condiciones extremas se tienen en cuenta en una serie de "índices de estrés" que estiman la amenaza que representa para la especie las condiciones de clima extremas o prolongadas de frío, calor y clima húmedo [16; 14]. Además, las limitaciones para finalizar el ciclo de vida debido a los períodos prolongados de insuficiente suma de calor o por un inadecuado régimen de horas luz pueden ser estimadas. Por último, los índices de crecimiento y estrés son combinados en un Índice Ecoclimático (IE), en una escala de 0 a 100, para representar la idoneidad de la ubicación geográfica para la propagación y persistencia de la especie. La variación en la idoneidad del clima para la sobrepoblación y crecimiento durante varios años también se pueden evaluar con CLIMEX utilizando la función Comparación de Años para Ceratitis capitata en el Ecuador en el año 2030.

El ajuste visual entre la distribución conocida y la distribución potencial actual de Ceratitis capitata se denomina proceso iterativo. Preliminarmente, se calibraron los parámetros de estrés y de humedad periódicamente, hasta que se represente la mejor aproximación entre la distribución potencial actual obtenida mediante CLIMEX versión 3 (formato ráster $18 \times 18 \mathrm{~km}^{2}$ ) y la distribución conocida de la especie (formato shapefile); ambos son exportados al programa ArcGis versión 10, para una mejor visualización. [17].

La determinación de clases del Índice Ecoclimático se realizó en el programa ArcGis ver. 10 (Proporties > Symbology > Quantities > 
Classify: Manual). Es decir el modelo está diseñado para imitar la integración de todas las influencias climáticas en las áreas de ubicación de Ceratitis capitata.

La distribución potencial actual de $C$. capitata.- Se realizó un registro de reportes de presencia y/o ocurrencia de Ceratitis capitata a nivel mundial, el cual se obtuvo por varias fuentes de información: publicaciones científicas, tesis, boletines especiales publicados por organismos oficiales de Agricultura de los diferentes países, revisiones bibliográficas generales, líneas de libre acceso, actas de congresos, etc.

Los datos se representaron en el programa Excel para tabularlos. Para cada uno de los puntos, de describió información como: país, primera, segunda y hasta tercera división administrativa del país, situación de la plaga, latitud, longitud, altitud, temperatura mínima de ambiente, temperatura máxima de ambiente, fechas de registro de captura, presencia o colecta de Ceratitis capitata, y referencias bibliográficas.

Cada lugar de colecta, captura y presencia fue referenciado, proporcionándosele datos de latitud y longitud (coordenadas geográficas) mediante dos herramientas: ArcGis versión $10 \mathrm{y}$ Google Earth versión 6.1. Las coordenadas fueron medidas en grados decimales y el datum en WGS 1984. El archivo Excel con (formato .xls) obtenido, se importó al programa ArcGis. A continuación se exportó el archivo Excel cambiado de formato .xls a formato .shp, obteniendose un shapefile de puntos de la distribución de Ceratitis capitata.

Validación de los resultados.- Se utilizaron datos de capturas de Ceratitis capitata, las mismas que se realizaron durante el año 2013 como producto de los monitoreos semanales en trampas que se implementaron en zonas estratégicas en el Ecuador.

La distribución potencial bajo el escenario de cambio climático A1B-2030.- Se realizó usando el escenario actual (1961-1990), el cual muestra un incremento en la temperatura promedio de $1.08^{\circ} \mathrm{C}$ a nivel global para el año 2030 [7].

En este caso, se utilizaron los datos meteorológicos como CLIMOND, a los que CLIMEX convierte en medias mensuales para afinar los datos e interpolar estos a valores semanales, que los hacen más comparables con los parámetros estimados. La función de comparación de años proporciona medidas relativas del crecimiento e índices de estrés que hacen posible la comparación entre estaciones y los años [16].
Los datos meteorológicos para condiciones actuales y futuras (Escenario A1B-2030) incluyen: temperatura mínima promedio mensual en ${ }^{\circ} \mathrm{C}$; temperatura máxima promedio mensual en ${ }^{\circ} \mathrm{C}$; precipitación promedio mensual en $\mathrm{mm}$; humedad relativa promedio mensual a las 9.00 horas y 15.00 horas respectivamente. [17].

Para determinar la distribución potencial mediante CLIMEX se usan dos metodologías complementarias. La primera es estimar la respuesta de las especies a la temperatura y la segunda es afinar los parámetros de CLIMEX con la distribución actual de la especie en un proceso iterativo [12]

\section{Resultados y discusión}

Distribución geográfica de Ceratitis capitata

A nivel mundial, se registraron 1376 datos de presencias de Ceratitis capitata, de las cuales 1067 registros presentan coordenadas espaciales y 309 registros son a nivel país o región. Los registros de la distribución de Moscamed pertenecen a 77 países distribuidos en los 5 continentes. El detalle de todos los registros se muestran en la Figura 1.

Respecto a los datos de presencias y ocurrencias para $C$. capitata; existen dos trabajos anteriores que en su metodología registran la georeferenciación del insecto a nivel mundial $[13 ; 16]$. En ambos casos, las bases de datos usadas registran datos de presencia de $C$. capitata para los 5 continentes; resultados que coinciden con los obtenidos en el presente estudio (Figura 1).

Szyniszewska y Tatem [13], usaron el modelo MaxEnt para realizar la evaluación global de estacionalidad de distribución potencial de Mosca del Mediterráneo, Ceratitis capitata, donde, se buscó en la literatura, la presencia histórica y contemporánea espacio-temporal de la plaga a nivel mundial. La base de datos construida contenía 2328 entradas geolocalizadas en sitios de detección de moscamed de 43 países y cerca de 500 localidades únicas (es decir, muchas georeferencias se repetían puesto que su interés fue estacional). Además, se añadió información sobre los hospederos, etapas de la vida y el método de captura. De estos, 125 localidades tenían información sobre el mes en que se registró la mosca mediterránea y estos datos se complementaron con material adicional de bases de datos completos disponibles en línea, en colecciones de museos, artículos publicados, informes y actas de congresos de presencia de $C$. capitata.

En cambio, Vera y otros [16] modelaron a $C$. capitata usando CLIMEX para determinar el 
potencial de distribución geográfica, haciendo énfasis en Argentina y Australia. El modelo fue utilizado para deducir las exigencias climáticas de Ceratitis capitata, usando como dato la distribución geográfica de moscamed en la Región mediterránea. Aunque en su investigación no especifican el número exacto de referencias usadas para el estudio, demostraron que esta plaga tiene un potencial de muy amplia distribución geográfica y que incluye zonas tropicales y subtropicales de todos los continentes, incluyendo aquellos con un clima mediterráneo.

\section{Parámetros proporcionados por el modelo CLIMEX para la elaboración del mapa de la distribución potencial de $\boldsymbol{C}$. capitata}

Los valores iniciales de los parámetros se establecieron según las recomendaciones de [16], pues se hizo necesario tomar valores de parámetros correspondientes a especies de clima tropical así como parámetros correspondientes a especies de clima templado, de tal manera que, los valores de los parámetros finales que se presenta en el cuadro 1 son representativos para especies tropicales $\mathrm{y}$ templadas, pues muestran el mayor ajuste de la distribución potencial en relación con la distribución observada.

Los parámetros de crecimiento, que corresponden a temperatura mínima óptima y temperatura máxima óptima fueron tomados de un estudio realizado por Vera [16].; la temperatura mínima limitante (DV0) fue tomada de Vargas y colaboradores [15], y la temperatura máxima limitante se tomó de Muniz, y colaboradores [9].
Los índices de humedad y los parámetros de estrés fueron determinados a partir del ajuste de la distribución potencial estimada por CLIMEX y la distribución conocida para $C$. capitata. Se ajustó el modelo CLIMEX para $C$. capitata, cambiando los parámetros iniciales (Cuadro 1), manualmente y de forma iterativa hasta que se determinó la distribución potencial (Valores del IE) para $C$. capitata; es decir, hasta que la distribución potencial actual coincida con la distribución geográfica de $C$. capitata (Cuadro 1). Dada la enorme variedad de posibles permutaciones estacionales de temperatura $\mathrm{y}$ humedad asociadas con localidades distribuidas en el espacio geográfico, el proceso de ajuste es, de hecho, muy exigente con el modelo [11].

\section{Distribución potencial actual de Ceratitis capitata para Ecuador}

Se hizo una proyección de la distribución potencial actual para el Ecuador, resultado de la interacción de los datos de presencia obtenidos a nivel mundial y los parámetros CLIMEX específicos para C. capitata.

En la Figura 3, Cuadro 2, se observa la distribución potencial actual de C. capitata para Ecuador, donde, los mayores porcentajes de superficie, presentan un IE $>30$, es decir que, el $47.73 \%$ del país tiene potencialmente condiciones favorables para que $C$. capitata se establezca todo el año; y un $47.61 \%$ de la superficie total en el Ecuador tiene condiciones menos favorables de establecimiento durante el año; en ambos casos, son áreas con condiciones favorables de presencia.

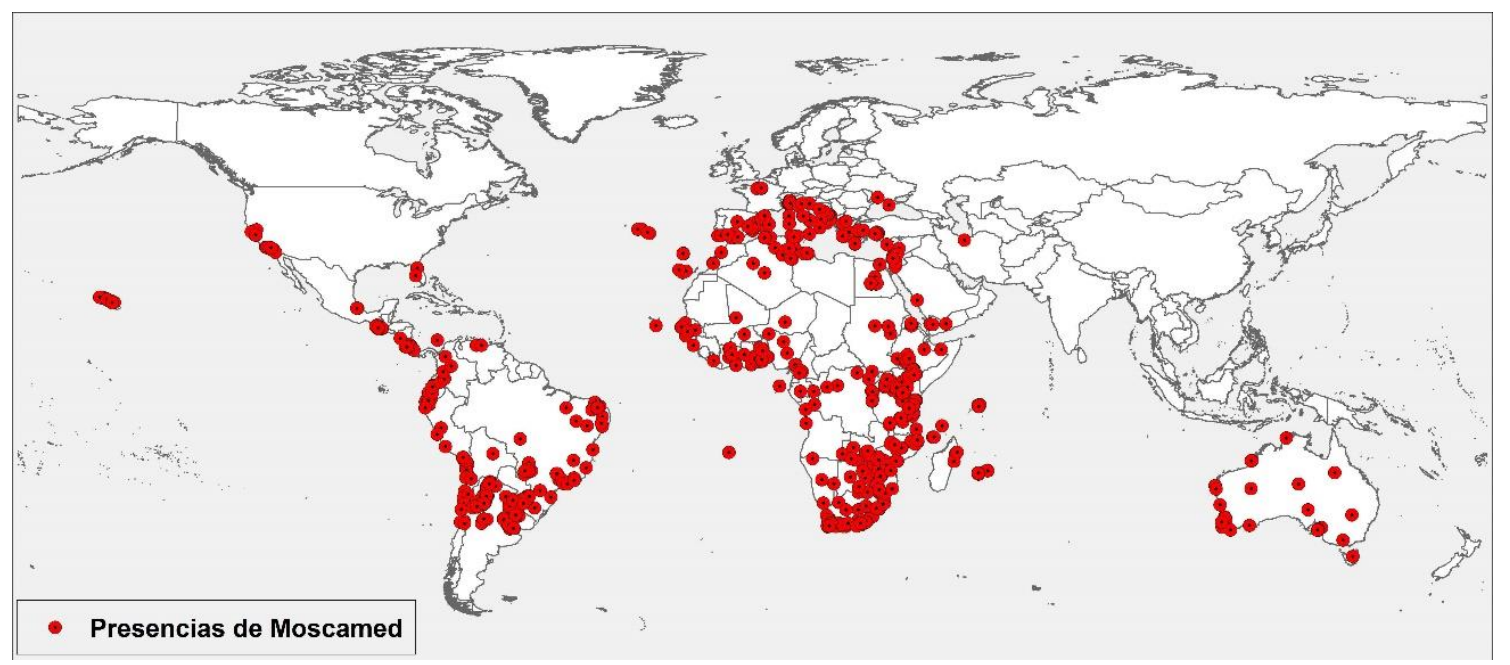

Figura 1.- Registros de presencia para Ceratitis capitata a nivel mundial 
Cuadro 1. Valores de los parámetros CLIMEX

\begin{tabular}{|c|c|c|c|}
\hline & $\begin{array}{l}\text { PARÁMETROS DE } \\
\text { CRECIMIENTO }\end{array}$ & $\begin{array}{c}\text { Valores } \\
\text { iniciales para } \\
\text { una especie } \\
\text { de clima } \\
\text { tropical }^{(\mathbf{a})}\end{array}$ & $\begin{array}{l}\text { Valores } \\
\text { finales }\end{array}$ \\
\hline \multicolumn{4}{|c|}{ Índices de Temperatura } \\
\hline DV0 & $\begin{array}{l}\text { Temperatura mínima } \\
\text { limitante }\left({ }^{\circ} \mathrm{C}\right) . \\
\text { Temperatura mínima }\end{array}$ & 8 & $9.5^{(\mathbf{b})}$ \\
\hline DV1 & $\begin{array}{l}\text { óptima }\left({ }^{\circ} \mathrm{C}\right) . \\
\text { Temperatura máxima }\end{array}$ & 16 & $22^{(\mathbf{c})}$ \\
\hline DV2 & $\begin{array}{l}\text { óptima }\left({ }^{\circ} \mathrm{C}\right) . \\
\text { Temperatura máxima }\end{array}$ & 28 & $24^{(\mathbf{c})}$ \\
\hline DV3 & limitante $\left({ }^{\circ} \mathrm{C}\right)$ & 32 & $35.09^{(\mathbf{d})}$ \\
\hline PDD & días-grados $\left(\mathrm{D}^{\circ}\right)$. & 400 & 280 \\
\hline \multicolumn{4}{|c|}{ Índices de humedad } \\
\hline SM0 & $\begin{array}{l}\text { del suelo }\left(\text { semana }^{-1}\right) \text {. } \\
\text { Humedad mínima óptima }\end{array}$ & 0.25 & 0 \\
\hline SM1 & $\begin{array}{l}\text { del suelo }\left(\text { semana }^{-1}\right) \\
\text { Humedad máxima óptima }\end{array}$ & 0.8 & 0.0051 \\
\hline SM2 & $\begin{array}{l}\text { del suelo }\left(\text { semana }^{-1}\right) \text {. } \\
\text { Humedad máxima limitante }\end{array}$ & 1.5 & 1.2789 \\
\hline SM3 & del suelo $\left(\right.$ semana $\left.^{-1}\right)$ & 2.5 & 1.631 \\
\hline
\end{tabular}

para la distribución potencial de $C$. capitata

$$
\begin{aligned}
& \text { (a) parámetros iniciales para especies de clima tropical } \\
& \text { según Sutherst y colaboradores [12] } \\
& \text { (b) Vargas y colaboradores [15] } \\
& \text { (c) Vera y colaboradores [16] } \\
& \text { (d) Muniz y Zalom [9] }
\end{aligned}
$$

El $4.65 \%$ de la superficie total tiene un $\mathrm{IE}=0$, pues en estas zonas, no es posible la presencia de moscamed debido a las condiciones climáticas adversas.

Las regiones costa, sierra y galápagos, siguen el mismo patrón (Figura3, Cuadro 2), donde el mayor porcentaje de sus áreas muestran un $\mathrm{IE}<30$, no así en la región Amazónica donde el $\mathrm{IE}<30$ es el que tiene el mayor porcentaje.

El modelo CLIMEX, se puede comparar con anteriores predicciones, tales como, la de De Meyer y otros [5], donde se usó un modelo de algoritmo genético para la predicción de un conjunto de reglas (GARP) y el análisis de componentes principales (PCA). Se usaron 8 covariables ambientales consistentes en parámetros de temperatura y precipitación junto a datos de ocurrencia de Moscamed en zonas nativas y no nativas. Para ambos casos (GARP y PCA), el autor muestra en sus resultados la presencia de moscamed en Ecuador, así como una posible distribución potencial tal como muestran también los resultados del presente estudio.

Vera y colaboradores [16] usaron CLIMEX georeferenciando la distribución geográfica de la región mediterránea mediante observaciones específicas sobre Sobrevivencia Invernal para predicciones que incorporaron el impacto del riego. Cuando la lluvia semanal fue inferior a 25 $\mathrm{mm}$, esta se incrementó a $25 \mathrm{~mm}$ mediante la opción apropiada en CLIMEX. Las predicciones del presente estudio no tomaron en cuenta el efecto del riego, pues la idea inicial es crear un mapa que sirva de preámbulo (línea base) para futuras investigaciones y como una segunda etapa de modelación con CLIMEX incorporar la adición de riego en determinadas zonas y épocas claves del año para Ecuador.

\section{Validación de las presencias de Ceratitis capitata en Ecuador}

Se implementaron una serie de trampas (Jackson y Mc Fail) en 8 provincias (Manabí, Guayas, Santa Elena, Carchi, Imbabura, Cotopaxí, Tungurahua, Azuay) del Ecuador durante el año 2013. Se registraron 611 datos de ocurrencias y/o presencias de Ceratitis capitata distribuidos en las 8 provincias del Ecuador. El detalle de todos los registros se muestran en la Figura 2; donde, se denota la coincidencia de zonas entre la distribución estimada resultante de aplicar el modelo CLIMEX y, la distribución conocida. Con esto se logró tener el mejor ajuste visual y asegurar la consistencia de los datos.

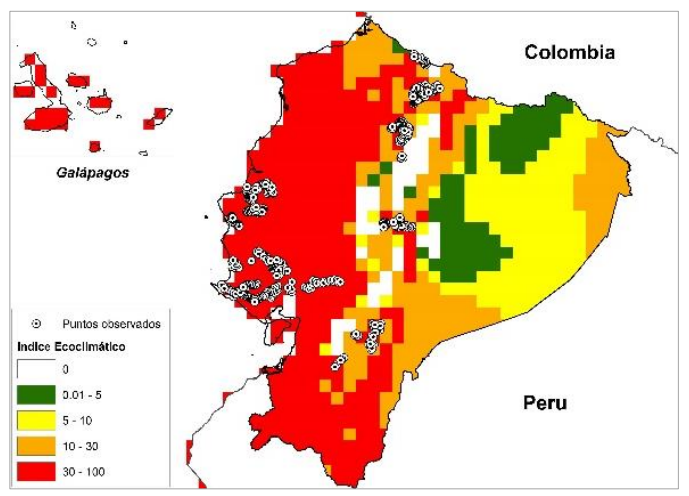

Figura 2.- Comparación entre la distribución observada de Ceratitis capitata con la distribución potencial actual proporcionada por CLIMEX para el Ecuador (ajuste visual). El color rojo (IE>30), indica zonas donde moscamed encuentra condiciones climáticas para establecerse durante todo el año.

\section{Distribución potencial bajo el escenario (A1B2030) de Ceratitis capitata}

El efecto del cambio climático bajo el escenario A1B2030 en la distribución de Ceratitis capitata para el Ecuador se presenta en la Figura 3, Cuadro 2, donde se pronostica que, el $62.17 \%$ de la superficie total presentará zonas menos favorables para que moscamed se establezca durante todo el año ( IE<30). 
Este dato está influenciado principalmente por los pronósticos para las regiones costa y oriente. El IE $>30$ representa un $33.03 \%$ de superficie a nivel país, y prevé mayores condiciones de establecimiento durante todo el año, resultado

influenciado principalmente por la predicción realizada para las regiones Insular (Galápagos) y Sierra.

Respecto a la diferencia en cambio de áreas entre el escenario actual y el escenario de cambio climático A1B2030 para Ceratitis capitata en Ecuador (Figura 3, Cuadro 2), se observa el porcentaje de superficie para cada predicción (actual y futura), donde, se estima que, en Ecuador, habrá una reducción de áreas idóneas para que moscamed se establezca durante todo el año (IE>30), resultado influenciado principalmente por la región costa donde se prevé que para el año 2030 el 39.12\% de la superficie tendrá condiciones climáticas para que $C$. capitata se establezca durante todo el año.

Se observa también que, las condiciones climáticas para la zona interior de la región costa, estarán sobre el Umbral de temperatura para estrés por calor que es de $35.1{ }^{\circ} \mathrm{C}$ y el Umbral de temperatura por exceso de calorhumedad que es de $35^{\circ} \mathrm{C}$ para $C$. capitata durante períodos del año (Cuadro 1), producto de la estimación en la variación global de temperatura en aumento de hasta $1.08^{\circ} \mathrm{C}$. Esta predicción, abre una posibilidad muy riesgosa para las regiones costa y sierra del País, pues el fenómeno descrito muy probablemente sea un factor que "obligue" a $C$. capitata a una migración o traslado hacia el perfil costanero causando su posible establecimiento durante todo el año en zonas donde solo se registraba ocurrencia y/o presencia.
Es posible también un desplazamiento hacia zonas de la sierra del Ecuador donde aún no existía moscamed o solo se registren ocurrencias y/o presencias, donde el cambio climático llegue a ser muy apto para el establecimiento.

Predicciones realizadas por Vera y colaboradores [17], muestran que, en ciertas zonas de Argentina como en Cerro Azul, se estima una reducción en el potencial de supervivencia en lugares húmedos, secos, o de prolongado estrés por calor durante períodos del año, lo que sugiere efectos perjudiciales de la humedad excesiva en el crecimiento probable de la población limitándose a determinados años.

Las zonas con un IE $=0$ en Ecuador en los escenarios actual y futuro (Figura 3, Cuadro 2), muestran que el umbral de temperatura para estrés por frío $\left(0^{\circ} \mathrm{C}\right)$ restringe la presencia de moscamed. Así mismo Vera, et al. 2002, mostraron resultados semejantes, ya que, en ciertas zonas de Argentina como en Jachal, el estrés por frío estuvo presente durante el invierno, y la combinación de ambos factores es probable que sea responsable de la baja abundancia de la plaga en dicha zona.

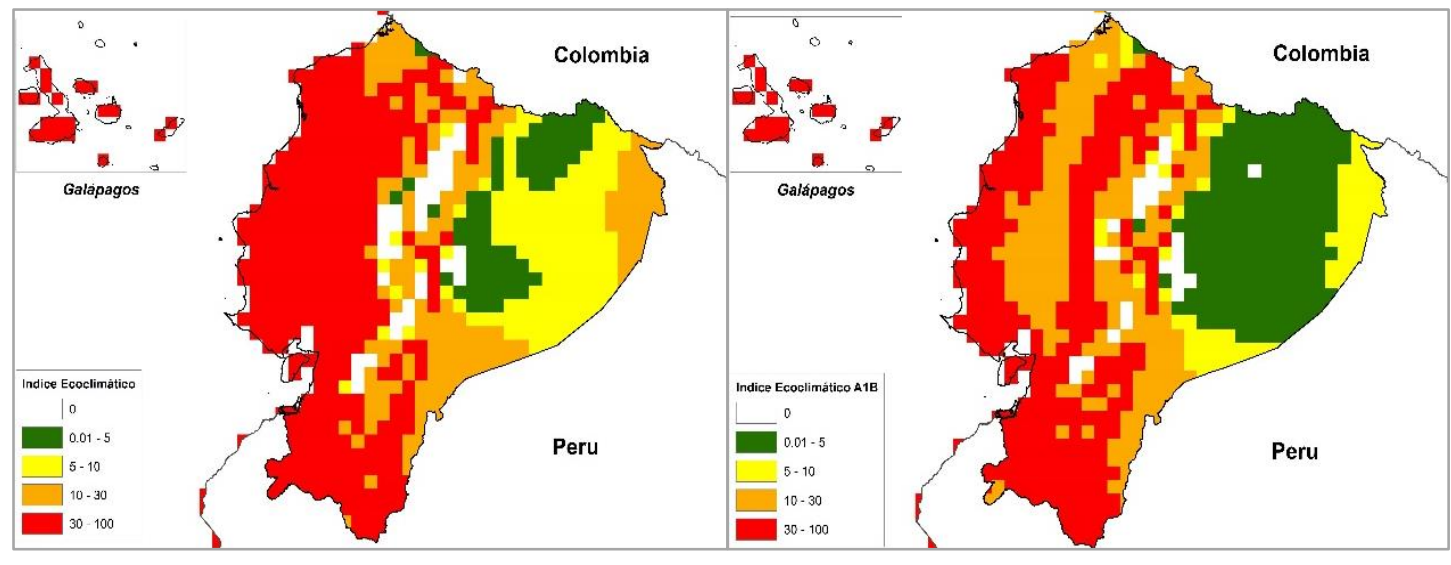

(a) (b)

Figura 3.- El mapa de la izquierda (a) representa la distribución potencial actual para Ceratitis capitata en el Ecuador, y el mapa de la derecha (b) representa la distribución potencial de Ceratitis capitata en el escenario de cambio climático A1B-2030 para el 
Cuadro 2.- Medida del cambio de áreas potenciales (\% de superficie) para Ceratitis capitata, en los escenarios actual y futuro A1B2030 para el Ecuador.

\begin{tabular}{|c|c|c|c|c|c|c|}
\hline \multirow{2}{*}{ Regiones } & \multicolumn{2}{|c|}{$\begin{array}{l}\text { IE }>30(\% \text { de la } \\
\text { superficie total) }\end{array}$} & \multicolumn{2}{c|}{$\begin{array}{c}\text { IE<30 (\% de la } \\
\text { superficie total) }\end{array}$} & $\begin{array}{c}\text { IE=0 (\% de la } \\
\text { superficie } \\
\text { total) }\end{array}$ \\
\cline { 2 - 8 } & Actual & $\begin{array}{c}\text { A1B - } \\
2030\end{array}$ & Actual & $\begin{array}{c}\text { A1B - } \\
2030\end{array}$ & Actual & $\begin{array}{c}\text { A1B - } \\
2030\end{array}$ \\
\hline Costa & 92.11 & 39.12 & 7.89 & 60.88 & 0 & 0 \\
Sierra & 57.04 & 57.83 & 30.13 & 34.5 & 12.83 & 7.67 \\
Oriente & 13.15 & 11.62 & 83.63 & 84.85 & 3.22 & 3.53 \\
Galápagos & 100 & 100 & 0 & 0 & 0 & 0 \\
\hline Total & 47.73 & 33.03 & 47.61 & 62.17 & 4.65 & 4.8 \\
\hline IE>30: zonas muy favorables (rojo), IE<30: zonas menos favorables \\
(naranja, amarillo y verde) e IE=0: zonas de probabilidades nulas \\
para establecimiento de C. capitata (blanco) durante todo el año.
\end{tabular}

El porcentaje de área de superficie varía muy poco en la región oriental comparando los escenarios: actual y A1B2030. Para ambos escenarios en el oriente, los mayores valores arrojan el IE <30, es decir que, estas zonas son menos favorables para que moscamed se establezca durante todo el año, debido a las altas precipitaciones observadas y estimadas en la zona que llegan entre 2000 a $4800 \mathrm{mmm}$ /año según el INAMHI [6].

Es una realidad que, aparte de las condiciones climáticas, existen otros factores que influyen en la presencia y establecimiento de Ceratitis capitata para el Ecuador, tales como la presencia y distribución de plantas hospederas, la presencia y distribución de especies competidoras como es el caso de Anastrephas y Bractoceras, los métodos de control de cobertura aplicados y patrones de movimiento y traslado del insecto (frutas, autos, etc). Por ello, trabajos como el presente representan un primer intento por predecir y modelar las zonas de distribución potenciales de moscamed en busca de enfoques alternativos para evitar pérdidas económicas, sociales y ambientales devastadoras en nuestros países.

\section{Conclusiones}

Se estimó la distribución potencial actual para C. capitata en el Ecuador usando el modelo CLIMEX. El $47.73 \%$ del país comprende zonas muy favorables (IE>30) para el establecimiento de $C$. capitata durante todo el año principalmente en la región costa. El $47.61 \%$ de la superficie total del país posee zonas menos favorables $(\mathrm{IE}<30)$ para la ocurrencia de $C$. capitata y el $4.65 \%$ del área del país comprende zonas con probabilidades nulas para el establecimiento y ocurrencia de moscamed $(\mathrm{IE}=0)$.

La estimación de la distribución potencial bajo un escenario de cambio climático (A1B-2030) para Ceratitis capitata en el Ecuador usando el modelo CLIMEX predijo que, el $33.03 \%$ del país corresponderán a zonas aptas con condiciones climáticas para que $C$. capitata se establezca todo el año (IE>30), el $62.17 \%$ de zonas del país posee zonas menos favorables para que $C$. capitata se establezca todo el año (IE<30), y en el $4.80 \%$ de zonas del país las probabilidades de establecimiento de $C$. capitata serán nulas ( $\mathrm{IE}=0)$.

\section{Referencias}

[1] AGROCALIDAD, 2013. Proyecto Nacional de Manejo de Moscas de la Fruta. Aprobado por Decreto Ejecutivo el 17 de abril del 2014. Ecuador. 107 p.

[2] APHIS, 1992. Risk assessment, Mediterranean fruit fly. Washington D.C.: Planning and Risk Analysis Systems. Policy and Program Development. Animal and Plant Health Inspection Service USDA.

[3] Carey JR., 1996. The Incipient Mediterranean Fruit Fly Population in California: Implications for Invasion Biology. Ecology 77: 1690-1697 doi:10.2307/2265775.

[4] Cross E., 2004. Mediterranean fruit flies attempt to sneak in-again. Cust Bord Prot Today.

[5] De Meyer M., Robertson M., Peterson A., Mansell M., 2008. Ecological niches and potential distribution of Mediterranean fruit fly (Ceratitis rosa). J Biogeogr 35: 270-281. Doi: 10.1111/j.1365-2699.2007.01769.x.

[6] INAMHI, 2013. Atlas hidrológico y metereológico estudiantil 2013. http://issuu.com/inamhi/docs/atlas_estudiantil

[7] Kriticos D., Webber B., Leriche A., Ota N., Macadam I., Bathols J., and Scott J., 2012. CliMond: global high-resolution historical and future scenario climate surfaces for bioclimatic modelling. Methods in Ecology and Evolution 3: 53-64.

[8] Liquido N., Cunningham R., Nakagawa S., 1990. Host Plants of Mediterranean Fruit Fly (Diptera: Tephritidae) on the Island of Hawaii (1949-1985 Survey). J Econ Entomol 83: 18631878.

[9] Muniz M., Zalom F., 1997. Developmental rate and number of generation estimates for Ceratitis capitata (Wiedemann) in fruit growing 
regions of California Bulletin OILB/SROP 20 55-66 USA, California. 15p.

[10]Siebert JB., Cooper T., 1995. If medfly infestation triggered a trade ban: Embargo on California produce would cause revenue, job loss. Calif Agric 49: 7-12 doi:10.3733/ca.v049n04p7.

[11] Sutherst G., 2003. Prediction of species geographical ranges. Journal of Biogeography 30: 805-816.

[12] Sutherst G., Maywald G., Kríticos D., 2007. CLIMEX version 3: user's guide. Australia, Hearne Scientific Software, CSIRO. $131 \mathrm{p}$.

[13] Szyniszewska, A. Tatem, A. 2014. Global Assessment of Seasonal Potential Distribution of Mediterranean Fruit Fly, Ceratitis capitata (Diptera: Tephritidae). PLoS ONE 9(11):e111582.

doi:10.1371/journal.pone.0111582

[14]Van Driesche R., Hoddle M., Center T., 2007. Control de Plagas y malezas por enemigos naturales. Trad. Ruíz, E; Coronada, J y Álvarez, J. Estados Unidos. 751 p.

[15] Vargas R., Walsh W., Jang E; Armstrongs J., and Kanehisa D., 1996. Survival and development of immature stages of four Hawaiian fruit flies (Diptera: Tephritidae) reared at five constant temperatures. Annals of the Entomological Society of America 89: 6469.
[16] Vera T., Rodriguez R., Segura D., Caldera J., Sutherst R., 2002. Potential Geographical Distribution of the Mediterranean Fruit Fly, Ceratitis capitata (Diptera: Tephritidae), with Emphasis on Argentina and Australia. Environmental Entomology. 31(6):1009-1022

[17]Veneros J., 2014. Distribución potencial de Phthorimaea operculella (Zeller), bajo condiciones del clima actual y un escenario de cambio climático. Tesis M.Sc. Ciencias Ambientales. Lima. Universidad Agraria La Molina. $152 \mathrm{p}$.

[18] Woodward F., 1988. Temperature and the distribution of plant species and vegetation. In: Long SP and Woodward FI (eds), Plants and Temperature. Society of Experimental Biology by The Company of Biologists Limited. Cambridge 42: 59-75 p. 